\title{
BIOPHYSICS
}

\section{A path to predict RNA tertiary structures}

\author{
A high-throughput platform allows biophysical measurements to probe RNA tertiary folding.
}

ntricate three-dimensional (3D) structures are critical for understanding RNA functions ranging from catalysis to pre-mRNA splicing. The hierarchical folding process from RNA sequence to secondary elements to tertiary structures is of great interest to RNA researchers.

Methods for predicting RNA secondary structures from a given sequence are available. Yet, it is still challenging to accurately predict the free energy of forming RNA tertiary structures owing to the $3 \mathrm{D}$ complexity.

William Greenleaf, Daniel Herschlag and their teams at Stanford University developed a thermodynamics-centric approach to study the sequence-structure-energy relationships in diverse RNA junctions. They leveraged the high-throughput capability of Illumina sequencing technology to quantitatively measure the binding equilibrium of tertiary contacts between a 'chip piece' that was immobilized on the surface and a 'flow piece' that contained the fluorophore for readout in solution. They investigated more than 1,000 RNA two-way junctions, unveiling principles of how changes in RNA sequences affect junction-assembly energetics.

These distinct thermodynamic profiles, or 'thermodynamic fingerprints', provide new insights into the rules governing the dynamic behaviors that are determined by the RNA sequence identity, the number and arrangement of unpaired residues, and the identity of noncanonical base pairs. Their findings support the concept of RNA 'ensemble modularity', by which the probability of tertiary-structure formation can be calculated through summation of sets of allowable structural conformations.
The high-throughput platform is adaptable to a variety of biomolecule binding studies, such as RNA-protein interactions. "Hopefully other researchers will use the dataset as a resource to learn about RNA structures that have not been crystallized," says Greenleaf. "In the long run, we hope to understand how RNA sequence influences functional tertiary structures both in vitro and in vivo."

Lei Tang

Published online: 31 August 2018 https://doi.org/10.1038/s41592-018-0133-9

Research papers

Denny, S. K. et al. High-throughput investigation of diverse junction elements in RNA tertiary folding. Cell 174, 377-390 (2018).

\section{We Create Solutions}

Ultra Precise Motion Control - D.C. Servo motors down to $20 \mathrm{~nm}$, piezos down to $1 \mathrm{~nm}$, and low drift XYZ stages.

Microscopy - Automation, modular microscopes, autofocus complete light sheet systems, and components. OEM - Custom designed systems to user specifications. 